\title{
ALTERAÇÕES FLORÍSTICAS OCORRIDAS NUM PERÍODO DE QUATORZE ANOS NA VEGETAÇÃO ARBÓREA DE UMA FLORESTA ESTACIONAL SEMIDECIDUAL EM VIÇOSA-MG ${ }^{1}$
}

\author{
Alessandro de Paula ${ }^{2}$, Alexandre Francisco da Silva ${ }^{3}$, Agostinho Lopes de Souza ${ }^{4}$ e \\ Flavio Antonio Maës dos Santos ${ }^{5}$
}

\begin{abstract}
RESUMO - Estudou-se um fragmento de Floresta Estacional Semidecidual Submontana, localizado no município de Viçosa (de 20 $35^{\circ}$ ' a $28^{\circ} 50^{\prime} \mathrm{S}$ e de $42^{\circ} 45^{\prime}$ a $43^{\circ} 00^{\prime} \mathrm{W}$ ), Estado de Minas Gerais, com os objetivos de identificar e analisar as alterações florísticas entre levantamentos realizados em 1984 e 1998. A listagem florística foi determinada a partir de um estudo fitossociológico no qual foi utilizado o método de parcelas contíguas, cobrindo uma área de 1 ha, tendo sido amostrados os indivíduos que apresentassem no mínimo $15 \mathrm{~cm}$ de CAP. Em 1998 foram encontradas 94 espécies, distribuídas em 77 gêneros e 32 famílias botânicas. Comparando os levantamentos de 1984 e 1998 , constatou-se que dez espécies entraram no perfil, sendo cinco secundárias tardias e cinco secundárias iniciais.
\end{abstract}

Palavras-chave: $\quad$ Mudanças florísticas, Floresta Estacional Semidecidual, composição florística e floresta mesófila.

\section{CHANGES IN THE FLORISTIC COMPOSITION OF WOOD SPECIES OBSERVED IN A 14 YEAR PERIOD IN A SEASONAL SEMIDECIDUOUS FOREST IN VIÇOSA-MG, SOUTHEASTERN BRAZIL}

\begin{abstract}
A fragment of Semideciduous Submontane Forest, located in Viçosa from (20'35' to 28 50'S and from $42^{\circ} 45^{\prime}$ to $\left.43^{\circ} 00^{\prime} W\right)$, Minas Gerais Southeastern Brazil, was studied to verify and analyze the changes that had occurred in its floristic composition between 1984 and 1998. All individuals with a trunk equal to or greater than $0.15 \mathrm{~m}$ of PBH of a phytosociological study were sampled in a grid plot of a hectare. In the 1998 survey, 94 species belonging to 77 genera and 32 families were sampled. Comparing the surveys of 1984 and 1998, it was observed that ten species were added to the experimental stand, with five of them being considered as initial secondary species and the other five as late secondary species.
\end{abstract}

Key words: $\quad$ Floristic changes, seasonal semideciduous forest, mesophitic Forest.

\section{INTRODUÇÃO}

A Zona da Mata de Minas Gerais era parte integrante de um continuum florestal de Mata Atlântica que, segundo Aubréville (1959), acompanhava o vale do rio Doce, desde o litoral do Estado do Espírito Santo até as proximidades de Belo Horizonte-MG.
No fim do século XIX e início do século XX, ocorreu o declínio da exploração de ouro em Mariana e Ouro Preto. A partir daí a cultura do café passou a expandir-se rapidamente pela região, sendo caracterizada principalmente pela monocultura predatória, pautada no desmatamento para o estabelecimento de novos plantios (Paniago, 1983).

1 Recebido para publicação em 30.5.2001.

Aceito para publicação em 3.12.2002.

Parte da Dissertação de Mestrado do primeiro autor - Bolsista da FAPEMIG.

2 Mestrado em Biologia Vegetal, pelo Dep. de Biologia Vegetal da Universidade Federal de Viçosa - UFV, 36571-000 ViçosaMG, <alessandrodepaula @ bol.com.br>; ${ }^{3}$ Prof. do Dep. de Biologia Vegetal da UFV; ${ }^{4}$ Prof. do Dep. de Engenharia Florestal da UFV. ${ }^{5}$ Prof. do Dep. de Botânica da Universidade de Campinas - UNICAMP, Caixa Postal 6109, 13081-970 Campinas-SP. 
Segundo Golfari (1975), as lavouras de café tornaram-se improdutivas rapidamente devido às características do solo (Latossolo Vermelho-Amarelo Distrófico, predominantemente) e do relevo (mares de morros), o que levou ao replantio de cafezais com plantas de novas procedências ou ao abandono do cultivo. Algumas dessas áreas foram ainda utilizadas para criação de gado em regime extensivo, o que dificultou ainda mais a regeneração natural das florestas.

O processo de ocupação antrópica, de modo geral, pode ser caracterizado pela substituição desordenada da cobertura vegetal original, pela abertura de estradas e acessos, pela implantação de lavouras, pastagens e reflorestamentos, como também pelo surgimento e crescimento de aglomerados urbanos. Dentro deste contexto, surgiram as formações vegetais secundárias, compostas por indivíduos resultantes de exploração seletiva ou de corte raso.

Gómez-Pompa \& Wiechers (1979) alertaram que, em um futuro próximo, os ecossistemas florestais primários serão exceções e estarão confinados em Unidades de Conservação. Assim, existe a necessidade de conhecer as fases sucessionais, não apenas para que se possa recuperar a vegetação original, mas também porque em cada fase se encontram potencialidades biológicas de grande utilidade para o homem, por exemplo os grupos de espécies de rápido crescimento, que podem ser exploradas comercialmente.

Segundo Tabarelli (1997), parte das transformações observadas na riqueza, diversidade, densidade de indivíduos e composição de espécies não é, a priori, direcional, convergente, progressiva ou organizada pela substituição de espécies ao longo do tempo ou pelo contínuo surgimento de propriedades emergentes. $\mathrm{O}$ autor ainda salientou que a substituição de espécies não é, necessariamente, o único processo responsável pela restauração da composição florística similar à da floresta nãoperturbada, caso ela ocorra.

Almeida \& Souza (1997) citaram que o desmatamento e a conseqüente fragmentação florestal podem provocar intensas mudanças na estrutura e no microclima da floresta, causando extinção local de espécies da flora e fauna.

Para Rankin-De-Merona \& Ackerly (1987), mesmo antes de completar o isolamento por desmatamento é possível constatar mudanças na composição de espécies do fragmento. Depois do isolamento, ocorrem também mudanças no microclima e na própria estrutura física do fragmento, as quais exercem influências sobre as espécies restantes e que podiam ser eventualmente refletidas em mudanças na composição taxonômica e demográfica. Uma espécie pode estar extinta anos antes da morte do último indivíduo, não sendo necessário nem que sua densidade populacional seja radicalmente reduzida para afetar o potencial reprodutivo. No caso de espécies dióicas, mudanças na razão sexual e a disponibilidade de polinizadores podem afetar a permanência da população na comunidade.

Viana (1990) considera que os efeitos da forma dos fragmentos sobre a diversidade biológica e sustentabilidade da floresta podem ser tão marcantes quanto os efeitos provocados pelo tamanho. Os fragmentos que possuem forma circular são menos influenciados pelo efeito de borda do que os alongados. Em fragmentos onde a relação borda/interior é alta, a composição das comunidades vegetais e animais deve apresentar menor diversidade.

Pretendeu-se analisar as alterações de ordem florística ocorridas entre dois levantamentos com intervalo de 14 anos, com o objetivo de contribuir para a compreensão da dinâmica sucessional em uma área de Floresta Estacional Semidecidual Submontana protegida permanentemente há 70 anos.

\section{MATERIAL E MÉTODOS}

A área de estudo situa-se em uma região pertencente às Terras Altas Brasileiras, numa divisão geopolítica conhecida como "Zona da Mata" de Minas Gerais, que segundo Rizzini (1963) faz parte da unidade fitogeográfica de Floresta Atlântica; para Veloso et al. (1991) ela faz parte da Floresta Estacional Semidecidual Montana. Neste trabalho concordou-se com a divisão de Oliveira-Filho et al. (1994), que fizeram uma modificação na proposta de Veloso et al. (1991) no limite altitudinal de 500 para $750 \mathrm{~m}$ entre as Formações Submontana e Montana, entre as latitudes de 16 e $24^{\circ}$ sul.

Segundo Valverde (1958), em suas condições originais a formação vegetal que sugeriu o nome "Zona da Mata" formava um contínuo com a floresta do médio Paraíba ao sul e do vale do rio Doce ao norte, sendo a oeste limitada pelos campos naturais do centro e do sul de Minas Gerais.

O município de Viçosa está localizado a 2045'S e $42^{\circ} 07^{\prime} \mathrm{W}$, a uma altitude de $650 \mathrm{~m}$, no sudeste do Estado 
de Minas Gerais, em região caracteristicamente montanhosa, cujo clima é do tipo Cwa ( mesotérmico, úmido com verões chuvosos e invernos secos), segundo a classificação de Köppen, apresentando déficit hídrico no período de maio a setembro e excedente de precipitação entre dezembro e março (Golfari, 1975). As médias anuais de precipitação pluvial, umidade relativa e temperatura do ar são, respectivamente, $1.340 \mathrm{~mm}, 80 \%$ e $19{ }^{\circ} \mathrm{C}$ (Castro et al., 1973).

A área de trabalho situa-se no campus da Universidade Federal de Viçosa (UFV), em um trecho conhecido como Mata da Biologia, que pertence a um fragmento de floresta do Jardim Botânico, possuindo uma área de aproximadamente 75 ha. No ano de 1922, a área foi totalmente desmatada, ocasião na qual foi implantado um cafezal. Com a fundação da Escola Superior de Agricultura e Veterinária (ESAV) em 1926, a cultura foi abandonada e a área passou a fazer parte do campus, permanecendo ter sob proteção permanente e em processo de regeneração natural até os dias atuais.

O trecho onde foi estabelecida a amostragem iniciase no sopé da encosta, chegando ao seu topo aos $730 \mathrm{~m}$ de altitude, apresentando declividade de até $45^{\circ}$ e face de exposição solar oeste-sudoeste.

A composição da lista florística limitou-se às espécies contempladas na amostragem fitossociológica de 1 hectare quadrado, no qual foram anotados todos os indivíduos de porte arbóreo que apresentassem circunferência à altura de 1,30 m do solo maior ou igual a $15 \mathrm{~cm}$ (CAP). Para apresentação da listagem florística foi adotado o sistema de Cronquist (1981), com exceção das famílias Caesalpiniaceae, Fabaceae e Mimosaceae, que foram mantidas como família Leguminosae e respectivas subfamílias. Os nomes das espécies, bem como seus autores, foram confirmados e atualizados pelo software do índice de espécies do ROYAL BOTANIC GARDEN (1993), ou por meio de literatura mais atualizada.

A determinação dos grupos ecológicos das espécies seguiu a proposta de Gandolfi et al. (1995).

O material-testemunho está depositado no Herbário do Departamento de Biologia Vegetal da Universidade Federal de Viçosa (VIC).

\section{RESULTADOS E DISCUSSÃO}

No presente trabalho foram amostradas 94 espécies, distribuídas em 77 gêneros e 32 famílias botânicas. Das famílias encontradas, 31 pertencem à classe Magnoliopsida e apenas uma, Arecaceae, é da classe Liliopsida, representada por uma única espécie, Attalea dubia Burret.

No Quadro 1 encontram-se listadas as espécies amostradas no levantamento fitossociológico realizado em 1998. Neste quadro observa-se que as mais ricas famílias foram: Leguminosae com 18, Lauraceae com nove, Myrtaceae com oito, Rubiaceae com seis, Flacourtiaceae com cinco, e Moraceae e Bignoniaceae com quatro cada uma.

Recorrendo a trabalhos desenvolvidos na Zona da Mata de Minas Gerais, como os de Almeida \& Souza (1997), Meira Neto et al. (1997), Marangon (1999), Meira Neto \& Martins (2000), Senra (2000), Silva et al. (2000) e Soares Júnior (2000), constatou-se nos levantamentos que nas Florestas Estacionais semideciduais da Zona da Mata de Minas Gerais as famílias Euphorbiaceae, Flacourtiaceae, Lauraceae, Leguminosae, Myrtaceae e Rubiaceae têm assumido importância relativa destacada, com especial contribuição à riqueza de espécies para a família Leguminosae, que não raramente se apresenta com o dobro do número de espécies da família que a segue em riqueza, enquanto a família Rubiaceae aumenta sua importância florística quando os critérios de inclusão nas amostragens são reduzidos, uma vez que muitas de suas espécies são características do sub-bosque.

Leitão Filho (1982) já havia destacado para as Florestas Estacionais Semideciduais do interior paulista a riqueza das famílias Leguminosae, Meliaceae, Rutaceae, Euphorbiaceae e Myrtaceae, além de Lauraceae, Rubiaceae e Moraceae.

Utilizando 11 levantamentos realizados na Floresta Ombrófila Densa (Atlântica) citados por Siqueira (1994), para a Região Sudeste, pôde-se observar que também nessas florestas a família Leguminosae se apresentou como a mais rica, seguida das famílias Lauraceae, Myrtaceae, Euphorbiaceae, Rubiaceae e Sapotaceae, respectivamente.

No presente levantamento, 14 famílias apresentaram apenas uma espécie, sendo elas: Anacardiaceae, Apocynaceae, Arecaceae, Burseraceae, Cecropiaceae, Celastraceae, Chrysobalanaceae, Erythroxylaceae, Nyctaginaceae, Phytolaccaceae, Rosaceae, Tiliaceae, Verbenaceae e Vochysiaceae.

O gênero mais rico foi Ocotea, com cinco espécies, seguido por Casearia com quatro e Eugenia com três. Sessenta e seis gêneros foram amostrados com apenas uma espécie, perfazendo $85,71 \%$.

R. Árvore, Viçosa-MG, v.26, n.6, p.743-749, 2002 
Quadro 1 - Espécies de porte arbóreo amostradas no levantamento fitossociológico realizado em 1998 na Mata da Biologia, campus da UFV, município de Viçosa - MG, apresentadas em ordem alfabética de famílias, gêneros e espécies, com os respectivos grupos ecológicos $(\mathrm{GE})$, em que $\mathrm{PI}=$ pioneira, $\mathrm{SI}=$ secundária inicial, $\mathrm{ST}$ = secundária tardia e $\mathrm{IND}=$ indiferente

Table 1 - Tree species sampled in 1998 year at the "Mata da Biologia" of the Federal University of Viçosa-MG, Southeastern Brazil, in alphabetical order of families, genera and species, followed by their respective ecological group $(G E)$. PI = pioneer, SI - initial secondary, ST - late secondary, IND - indifferent

\begin{tabular}{|c|c|c|}
\hline Família & Espécie & $\mathrm{GE}$ \\
\hline Anacardiaceae & Astronium fraxinifolium Schott & ST \\
\hline \multirow{2}{*}{ Annonaceae } & Rollinia silvatica Mart. & SI \\
\hline & Xylopia sericea A. St.-Hil. & SI \\
\hline Apocynaceae & Aspidosperma olivaceum Müll. Arg. & ST \\
\hline Arecaceae & Attalea dubia Burret & PI \\
\hline \multirow{4}{*}{ Bignoniaceae } & Cybistax antisyphilitica Mart. & SI \\
\hline & Jacaranda macrantha Cham. & SI \\
\hline & Sparattosperma leucanthum K.Schum. & SI \\
\hline & Zeyheria tuberculosa Bur. ex Verlot & ST \\
\hline \multirow{2}{*}{ Bombacaceae } & Ceiba speciosa (A.St.-Hil.) Gibbs et Semir & SI \\
\hline & Eriotheca candolleana (K. Schum.) A. Robyns & SI \\
\hline \multirow{2}{*}{ Boraginaceae } & Cordia sellowiana Cham. & SI \\
\hline & Cordia sylvestris Fresen. & SI \\
\hline Burseraceae & Protium warmingianum March. & ST \\
\hline Cecropiaceae & Cecropia hololeuca Miq. & PI \\
\hline Celastraceae & Maytenus aquifolium Mart. & ST \\
\hline Chrysobalanaceae & Licania spicata Hook. f. & SI \\
\hline Erythroxylaceae & Erythroxylum pelleterianum A. St.-Hil. & SI \\
\hline \multirow{3}{*}{ Euphorbiaceae } & Croton floribundus Spreng. & PI \\
\hline & Mabea fistulifera Mart. & PI \\
\hline & Sapium glandulatum (Vell.) Pax & PI \\
\hline \multirow{5}{*}{ Flacourtiaceae } & Carpotroche brasiliensis Endl. & ST \\
\hline & Casearia arborea Urb. & SI \\
\hline & Casearia decandra Jacq. & SI \\
\hline & Casearia obliqua Spreng. & SI \\
\hline & Casearia ulmifolia Cambess. & SI \\
\hline \multirow{9}{*}{ Lauraceae } & Aniba firmula Mez & ST \\
\hline & Endlicheria paniculata (Spreng.) J.F. Macbr. & ST \\
\hline & Nectandra lanceolata Ness et Mart. ex Nees & ST \\
\hline & Ocotea dispersa Mez & ST \\
\hline & Ocotea indecora Schott ex Meissn. & ST \\
\hline & Ocotea odorifera (Vell.) Rohwer & ST \\
\hline & Ocotea pubescens Mez & ST \\
\hline & Ocotea pulchella Mart. & SI \\
\hline & Persea pyrifolia Nees & SI \\
\hline \multirow{2}{*}{ Lecythidaceae } & Cariniana estrellensis Kuntze & ST \\
\hline & Cariniana legalis Kuntze & ST \\
\hline \multirow{3}{*}{ Leguminosae-Caesalpinioideae } & Apuleia leiocarpa J.F. Macbr. & SI \\
\hline & Copaifera langsdorffii Desf. & SI \\
\hline & Peltophorum dubium Taub. & SI \\
\hline \multirow{5}{*}{ Leguminosae-Faboideae } & Andira fraxinifolia Benth. & SI \\
\hline & Dalbergia nigra Allemão ex Benth. & SI \\
\hline & Lonchocarpus guilleminianus (Tul.) Malme & SI \\
\hline & Machaerium brasiliense Vogel & SI \\
\hline & Machaerium nyctitans (Vell.) Benth. & PI \\
\hline \multicolumn{3}{|c|}{$\begin{array}{l}\text { Continua. } \\
\text { Continued. }\end{array}$} \\
\hline
\end{tabular}


Quadro 1, cont.

Table 1, cont.

\begin{tabular}{|c|c|c|}
\hline Família & Espécie & $\mathrm{GE}$ \\
\hline \multirow{6}{*}{ Leguminosae-Faboideae } & Myroxylon peruiferum L.f. & ST \\
\hline & Platymiscium pubescens Micheli & ST \\
\hline & Platypodium elegans Vogel. & SI \\
\hline & Pterocarpus rohrii Vahl & SI \\
\hline & Swartzia elegans Schott & ST \\
\hline & Zollernia ilicifolia Vogel. & ST \\
\hline \multirow{4}{*}{ Leguminosae-Mimosoideae } & Acacia polyphylla DC. & SI \\
\hline & Anadenanthera colubrina (Vell.) Brenan & SI \\
\hline & Inga striata Benth. & SI \\
\hline & Piptadenia gonoacantha J.F. Macbr. & PI \\
\hline \multirow{3}{*}{ Meliaceae } & Cedrela fissilis Vell. & SI \\
\hline & Trichilia elegans A. Juss. & ST \\
\hline & Trichilia pallida $\mathrm{Sw}$. & SI \\
\hline \multirow{2}{*}{ Monimiaceae } & Mollinedia argyrogyna Perkins & SI \\
\hline & Siparuna guianensis Aubl. & IND \\
\hline \multirow{4}{*}{ Moraceae } & Acanthinophyllum ilicifolium (Spreng.) Burger & SI \\
\hline & Brosimum glaziovii Taub. & SI \\
\hline & Maclura tinctoria D. Don ex Steud. & SI \\
\hline & Sorocea bonplandii (Baill.) Burger, Lanj. et Boer & SI \\
\hline \multirow{8}{*}{ Myrtaceae } & Campomanesia xanthocarpa Berg & ST \\
\hline & Eugenia leptoclada Berg & ST \\
\hline & Eugenia stictosepala Kiaersk. & $\mathrm{ST}$ \\
\hline & Eugenia uniflora L. & ST \\
\hline & Myrcia fallax DC. & SI \\
\hline & Myrcia sphaerocarpa DC. & SI \\
\hline & Myrciaria axillaris Berg & SI \\
\hline & Plinia glomerata (Berg) Amshoff & SI \\
\hline Nyctaginaceae & Guapira opposita (Vell.) Reitz & SI \\
\hline Phytolaccaceae & Seguieria americana $\mathrm{L}$. & SI \\
\hline Rosaceae & Prunus sellowii Koehne & SI \\
\hline \multirow{6}{*}{ Rubiaceae } & Amaioua guianensis Aubl. & SI \\
\hline & Bathysa nicholsonii $\mathrm{K}$. Schum. & SI \\
\hline & Coutarea hexandra (Jacq.) K. Schum. & SI \\
\hline & Guettarda viburnoides Cham. et Schltdl. & SI \\
\hline & Ixora gardneriana Benth. & ST \\
\hline & Psychotria carthagenensis Jacq. & SI \\
\hline \multirow{2}{*}{ Rutaceae } & Hortia arborea Engl. & ST \\
\hline & Zanthoxylum rhoifolium Lam. & PI \\
\hline \multirow{3}{*}{ Sapindaceae } & Allophylus edulis Radlk. ex Warm. & SI \\
\hline & Matayba elaeagnoides Radlk. & SI \\
\hline & Matayba guianensis Aubl. & SI \\
\hline \multirow{3}{*}{ Sapotaceae } & Chrysophyllum gonocarpum Engl. & ST \\
\hline & Chrysophyllum marginatum Radlk. & $\mathrm{ST}$ \\
\hline & Pouteria laurifolia Radlk. & $\mathrm{ST}$ \\
\hline \multirow{2}{*}{ Simaroubaceae } & Picramnia regnelli Engl. & SI \\
\hline & Picramnia sp. & SI \\
\hline Tiliaceae & Luehea grandiflora Mart. & PI \\
\hline Verbenaceae & Vitex megapotamica (Spreng.) Moldenke & SI \\
\hline Vochysiaceae & Qualea jundiahy Warm. & $\mathrm{ST}$ \\
\hline
\end{tabular}


Em 1984 foram encontradas 90 espécies distribuídas em 77 gêneros e 34 famílias botânicas (Silva et al., 2000). Dessas famílias, 35 são da classe Magnoliopsida e apenas uma, Arecaceae, é da classe Liliopsida, representada por uma única espécie, Attalea dubia.

Das famílias amostradas em 1984, apenas duas, Guttiferae e Olacaceae, não foram encontradas em 1998. Guttiferae era representada pelas espécies Rheedia gardneriana Planch. et Triana e Tovomitopsis saldanhae Engl., pertencentes aos grupos das secundárias iniciais e tardias, respectivamente. Já Olacaceae foi representada por uma única espécie, Citronella paniculata (Mart.) Howard, secundária inicial.

Apenas a família Boraginaceae teve o número de espécies reduzido em 1998, passando de quatro para duas. As espécies que saíram da amostra foram Cordia bullata Roem. et Schult. e Cordia magnoliaefolia Cham., ambas secundárias iniciais.

Outra família que apresentou modificações foi Rubiaceae. A espécie Psychotria sessilis Müll. Arg., secundária inicial, não foi encontrada no levantamento de 1998. Em compensação, Psychotria carthagenensis, também secundária inicial, foi amostrada apenas em 1998.

Dentre as famílias com maior número de espécies, três aumentaram sua representatividade. Leguminosae passou de 16 para 19 espécies com a entrada de Inga striata, Machaerium brasiliense, secundárias iniciais, e Platymiscium pubescens, secundária tardia. Lauraceae passou de seis para nove espécies, sendo as recém-ingressadas: Nectandra lanceolata, Ocotea indecora e Ocotea pubescens, todas secundárias tardias. Myrtaceae passou de seis para oito espécies com a entrada de Eugenia uniflora, secundária tardia, e de Myrcia fallax e Simaroubaceae, que ingressou no perfil com Picramnia sp., sendo essas duas últimas espécies secundárias iniciais.

Das dez espécies que entraram no perfil, cinco são secundárias tardias e as outras cinco são secundárias iniciais. Vale ressaltar que nenhuma espécie pioneira diferente das listadas em 1984 foi encontrada em 1998.

Outro fato importante foi que das seis espécies que saíram do perfil, cinco são secundárias iniciais e apenas uma, Tovomitopsis saldanhae, é secundária tardia.

\section{CONCLUSÕES}

Os dados demonstraram que o atual estádio sucessional do fragmento tem favorecido o estabelecimento de espécies tardias, em detrimento das iniciais (pioneiras e secundárias iniciais), denotando uma evolução sucessional no intervalo entre os dois levantamentos considerados, o que é esperado em fragmentos eficientemente preservados.

Confirmou-se a importância das famílias Euphorbiaceae, Flacourtiaceae, Lauraceae, Leguminosae, Myrtaceae e Rubiaceae para as Florestas Estacionais Semideciduais da Zona da Mata de Minas Gerais, com destaque para Leguminosae, assim como sua importância para a mesma Formação no Planalto Paulista e para as florestas Ombrófilas Densas da encosta atlântica, destacando a unidade fitogeográfica da Floresta Atlântica, sensu Rizzini (1963).

\section{REFERÊNCIAS BIBLIOGRÁFICAS}

ALMEIDA, D. S.; SOUZA, A. L. Florística e estrutura de um fragmento de Floresta Atlântica, no município de Juiz de Fora, Minas Gerais. Revista Árvore, v. 21, n. 2, p. 221-230, 1997.

AUBRÉVILLE, A. As florestas do Brasil - estudo fitogeográfico florestal. Anuário Brasileiro de Economia Florestal, n. 11, p. 201-232, 1959.

CASTRO, P. S. et al. Interceptação da chuva por mata natural secundária na região de Viçosa, MG. Revista Árvore, v. 7, p. 76-88, 1973.

CRONQUIST, A. The evolution and classification of flowering plants. New York: The New York Botanical Garden, 1981. 555 p.

GANDOLFI, S.; LEITÃO FILHO, H. F.; BEZERRA, C. L. F. Levantamento florístico e caráter sucessional das espécies arbustivo-arbóreas de uma floresta mesófila semidecídua no município de Guarulhos, SP. Revista Brasileira de Biologia, v. 55, n. 4 , p. $753-767,1995$.

GOLFARI, L. Zoneamento ecológico do Estado de Minas Gerais para reflorestamento. Belo Horizonte: PRODEPEF/ PNUD/FAO/IBDF/Bra-45, 1975. 65 p. (Série técnica, 3).

GÓMEZ-POMPA, A.; WIECHERS, B. L. Regeneracion de los ecossistemas tropicales y subtropicales. In: GÓMEZPOMPA, A.; AMO, R. S. (Eds.). Investigaciones sobre la regeneración de las selvas altas en Vera Cruz, México. México: Companhia Editorial Continental, 1979. p. 11-30.

LEITÃO FILHO, H. F. Aspectos taxonômicos das florestas do estado de São Paulo. In: CONGRESSO NACIONAL SOBRE ESSÊNCIAS NATIVAS, 1982, São Paulo. Anais... São Paulo: Silvicultura em São Paulo, v. 16A, pt. 1, p. 197206, 1982. 
MARANGON, L. C. Florística e fitossociologia de área de floresta estacional semidecidual visando dinâmica de espécies florestais arbóreas no município de Viçosa, MG. São Carlos: UFSCAR, 1999. 135 p. Tese (Doutorado em Ecologia e Recursos Naturais) - Universidade Federal de São Carlos, 1999.

MEIRA NETO, J. A. A. et al. Estrutura de uma Floresta Estacional Semidecidual Submontana em área diretamente afetada pela Usina Hidrelétrica de Pilar, Ponte Nova, Zona da Mata de Minas Gerais. Revista Árvore, v. 21, n. 3, p. 337-344, 1997.

MEIRA NETO, J. A. A.; MARTINS, F. R. Estrutura da Mata da Silvicultura, uma floresta estacional semidecidual Montana no município de Viçosa, MG. Revista Árvore, v. 24, n. 2, p. 151-160, 2000.

OLIVEIRA-FILHO, A. T.; SCOLFORO, J. R. S.; MELLO, J. M. Composição florística e estrutura comunitária de um remanescente de floresta semidecídua montana em Lavras, MG. Revista Brasileira de Botânica, v. 17, n. 2, p. 167182, 1994.

PANIAGO, M. C. T. Evolução histórica e tendências de mudanças sócio-culturais na comunidade de Viçosa - MG Viçosa-MG, UFV, 1983. 407 p. Dissertação (Mestrado em Economia Rural) - Universidade Federal de Viçosa, 1983.

RANKIN-DE-MERONA, J. M.; ACKERLY, D. D. Estudos populacionais de árvores em florestas fragmentadas e as implicações para conservação in situ das mesmas na floresta tropical da Amazônia Central. Revista IPEF, n. 35, p. 47$59,1987$.

RIZZINI, C. T. Nota prévia sobre a divisão fitogeográfica (florístico-sociológica) do Brasil. Revista Brasileira de Geografia, v. 25, n. 1, p. 3-64, 1963.

ROYAL BOTANIC GARDEN. Index Kewensis on compact disc - manual. Oxford: Oxford University Press, 1993. $67 \mathrm{p}$.
SENRA, L. C. Composição florísrica e estrutura fitossociológica de um fragmento florestal da Fazenda Rancho Fundo, Zona da Mata - Viçosa, MG . Viçosa-MG: UFV, 2000. 66 p. Dissertação (Mestrado em Botânica) Universidade Federal de Viçosa, 2000.

SILVA, A. F.; FONTES, N. R. L.; LEITÃO FILHO, H. F. Composição florística e estrutura horizontal do estrato arbóreo de um trecho da Mata da Biologia da Universidade Federal de Viçosa - Zona da Mata de Minas Gerais. Revista Árvore, v. 24, n. 4, p. 397-406, 2000.

SIQUEIRA, M. F. Análise florística e ordenação de espécies arbóreas da Mata Atlântica através de dados binários. Campinas: UNICAMP, 1994. 143 p. Dissertação (Mestrado em Biologia Vegetal) - Universidade Estadual de Campinas, 1994.

SOARES JÚNIOR, F. J. Composição florística e estrutura de um fragmento de Floresta Estacional Semidecidual na Fazenda Tico-Tico, Viçosa, MG. Viçosa: UFV, 2000. 68 p. Dissertação (Mestrado em Botânica) - Universidade Federal de Viçosa, 2000.

TABARELLI, M. A regeneração da floresta atlântica montana. São Paulo: USP, 1997. 104 p. Tese (Doutorado em Ecologia) - Universidade de São Paulo, 1997.

VALVERDE, O. Estudo regional da Zona da Mata, de Minas Gerais. Revista Brasileira de Geografia, v. 20, n. 1, p. 3-82, 1958.

VELOSO, H. P.; RANGEL FILHO, A. L. R.; LIMA, J. C. A. Classificação da vegetação brasileira, adaptada a um sistema universal. Rio de Janeiro: IBGE, 1991. 123 p.

VIANA, V. M. Biologia e manejo de fragmentos florestais naturais. In: CONGRESSO FLORESTAL BRASILEIRO, 6., 1990, Campos do Jordão. Anais... Campos do Jordão: 1990. v. 1, p. 113-118. 